\title{
Polyion Adsorption onto Catanionic Surfaces. A Monte Carlo Study
}

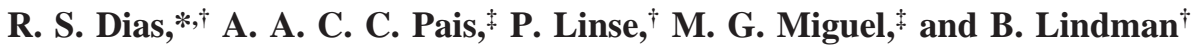 \\ Physical Chemistry 1, Center for Chemistry and Chemical Engineering, Lund University, P.O. Box 124, \\ S-221 00 Lund, Sweden, and Departamento de Química, Universidade de Coimbra, \\ 3004-535 Coimbra, Portugal
}

Received: January 10, 2005; In Final Form: April 7, 2005

\begin{abstract}
The adsorption of a single and negatively charged polyion with varying flexibility onto a surface carrying both negative and positive charges representing a charged membrane surface has been investigated by using a simple model employing Monte Carlo simulations. The polyion was represented by a sequence of negatively charged hard spheres connected with harmonic bonds. The charged surface groups were also represented by charged hard spheres, and they were positioned on a hard surface slightly protruding into the solution. The surface charges were either frozen in a liquidlike structure or laterally mobile. With a large excess of positive surface charges, the classical picture of a strongly adsorbed polyion with an extended and flat configuration emerged. However, adsorption also appeared at a net neutral surface or at a weakly negatively charged surface, and at these conditions the adsorption was stronger with a flexible polyion as compared to a semiflexible one, two features not appearing in simpler models containing homogeneously charged surfaces. The presence of charged surface patches (frozen surface charges) and the ability of polarization of the surface charges (mobile surface charges) are the main reasons for the enhanced adsorption. The stronger adsorption with the flexible chain is caused by its greater ability to spatially correlate with the surface charges.
\end{abstract}

\section{Introduction}

Polymer and protein adsorption onto lipid monolayers and bilayers is of fundamental importance in biology as well as in a large range of technological processes, such as pharmacology. For example, the adsorption of macromolecules onto surfaces of substrates is an intermediate step in fabrication of drug and gene delivery vehicles.

One of the most studied systems of nonviral gene therapy consists of the so-called lipoplexes, complexes formed between DNA molecules and liposomes (vesicular structures formed typically by a mixture of a neutral and a cationic lipid). ${ }^{1,2}$ The formation of such complexes starts with the adsorption of DNA onto the positively charged membrane. These systems have been extensively studied, and even though the mechanism of formation is still far from understood, ${ }^{3,4}$ the structure of the complexes is believed to be a short-ranged lamellar structure composed of amphiphilic bilayers with DNA molecules ordered and packed between the lipid stacks. This type of structure has been observed for systems with different lipid components. ${ }^{5-8}$ Moreover, DNA with its unique structure can also act as a good candidate for future nanodevices such as templates, biosensors, and semiconducting molecules.

There are a significant number of experimental studies on the adsorption of polyelectrolytes, especially DNA, on liposomes composed of neutral and positively charged lipids (for reviews, see refs 2 and 9-11). Ellipsometry studies have shown that a thick layer of a few DNA molecules adsorbed on a hydrophobic surface undergoes strong condensation into a thin and denser layer by the addition of a cationic surfactant. ${ }^{12,13}$ DNA adsorption on cationic lipid bilayers was also studied by atomic force microscopy, and the large DNA molecules were shown to

* Corresponding author.

$\dagger$ Lund University.

$\doteqdot$ Universidade de Coimbra. destabilize the membrane. ${ }^{14,15}$ The adsorption has also been followed by surface plasmon spectroscopy ${ }^{16}$ and fluorescence microscopy, and it was found that the molecules, when confined in two dimensions, adsorb in an extended conformation. ${ }^{17,18}$

Specific studies on the adsorption of polyelectrolytes on vesicles formed by mixtures of positively and negatively charged surfactants are more scarce. ${ }^{8,19-21}$ It was observed that DNA, in general, destroys the vesicles, ${ }^{8}$ whereas more flexible macromolecules induce changes in the shapes of the vesicles. ${ }^{19,21,22}$ However, for minute concentrations of DNA, most of the vesicles were intact, and the interaction caused a certain degree of compaction in the polymer backbone. ${ }^{23}$ Figure 1 illustrates the experimental observation of a single DNA chain adsorbing upon a catanionic vesicle. There is some indication that the polymer chain has a tendency to reside at the surface of the vesicle, but the underlying adsorption mechanism or the disruption caused to the surface calls for rationalization.

When a lamellar phase is in its fluid state, the lipids possess relatively fast lateral diffusion, which are, in principle, responsive when a charged object approaches. In fact, this demixing of the lipids and formation of domains in mixed lipid membranes have been observed recently by fluorescence microscopy experiments upon the adsorption of DNA on cationic membranes $^{18}$ or of peptides on giant unilamellar vesicles. ${ }^{24}$ In biological membranes, the domains formed are often denoted as "rafts". The rapid lateral movement of lipid molecules, or membrane fluidity, is believed to be responsible for the proper functioning of several membrane properties. This mobility of charges in the plane can lead to interesting properties. Indeed, calculations have shown that the membrane fluidity has a considerable influence on DNA adsorption, ${ }^{25,26}$ even leading to the counterintuitive phenomenon of a negatively charged polymer adsorbing on an overall negatively charged surface. ${ }^{26}$ However, atomic force experiments on the DNA interaction with 


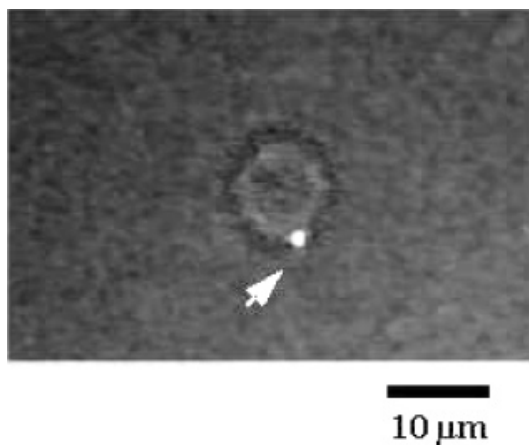

Figure 1. Optical microscopy image of a single T4DNA globule adsorbed onto the surface of a positively charged catanionic vesicle. The arrowhead indicates a single DNA globule. Reproduced from ref 23 .

homogeneous and overall neutral lipid bilayers, composed of cationic and anionic lipids, with no fluidity, show no adsorption. It was also shown, not surprisingly, that the DNA adsorption is dependent on the surface charge density and on the size of the cationic patches in the lipid bilayers. ${ }^{27}$

Because of the obvious interest in this field, the adsorption of a polymer onto substrates has also been the subject of many theoretical and simulation studies (for review, see refs 28-30). Studies on the adsorption of a charged polymer onto an oppositely charged homogeneous surface are the most abundant, and the phenomena involved are reasonably well-understood. Recently, some molecular simulation studies on the adsorption of macromolecules on heterogeneous surfaces with patches of different charges and charge densities ${ }^{31-33}$ and theoretical studies involving heterogeneous or "fluid" surfaces ${ }^{25,26,34-36}$ show a clear attempt to mimic real systems.

The aim of the present study is to increase the complexity of the description of the membrane surface to discriminate the roles of different components of the model, yet using a simple model. Here, our focus is on how the fluidity of a surface containing both positive and negative charges affects the adsorption of a flexible and semiflexible negatively charged polyion in a saltfree solution. Two important findings are that (i) adsorption appears even onto a net neutral surface or a weakly net negatively charged surface and (ii) there are conditions at which a flexible polyion adsorbs more strongly than a more rigid one.

\section{Model and Method}

2.1. Model. A simple model has been adopted to describe the adsorption of polyions from solution onto charged surfaces carrying both cations and anions. Important aspects of the model include (i) a generic spring-bead chain representing the polymer, (ii) surface groups protruding into the solution, and (iii) an explicit description of all charged species.

The negatively charged polyion (polyanion) is described as a sequence of negatively charged hard spheres (beads) connected with harmonic bonds with the chain flexibility regulated by angular force terms. The charged surface is composed of a hard planar wall with embedded positively and negatively charged hard spheres. Conditions representing two membrane fluidities are considered: surface charges with fixed positions (referred to as the frozen condition) and laterally mobile surface charges (referred to as the mobile condition). In addition, a minimum amount of monovalent simple ions, also treated as charged hard spheres, is included to obtain an electroneutral system. The solvent enters the model only through its relative permittivity.

Consider a rectangular box with box lengths $L_{x}=L_{y}=200$ $\AA$ and $L_{z}=400 \AA$. Hard walls are placed at $z=z_{\text {wall }}=-150$

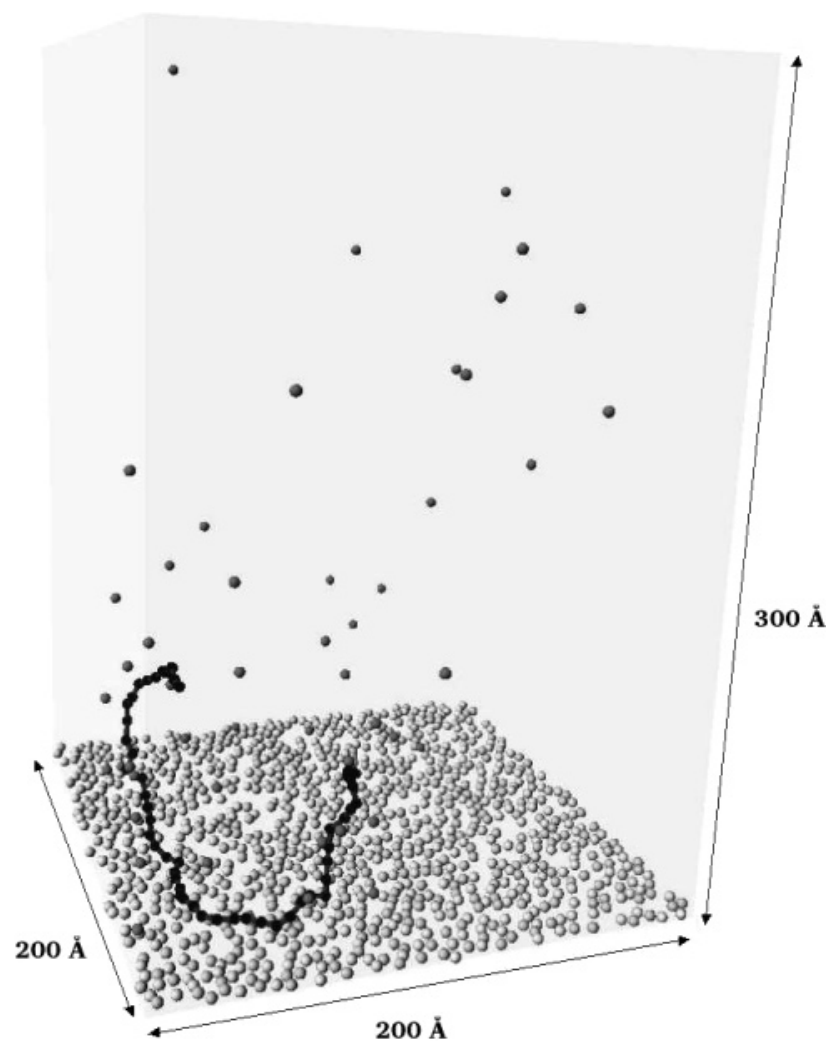

Figure 2. Graphic representation of an aqueous solution containing one polyion and simple ions in contact with a catanionic surface. Here, the net charge of the surface is zero and the simple ions are thus counterions to the polyion. The dimensions of the solution volume are also displayed. All particles have a hard-sphere radius $R=2 \AA$.

and $150 \AA$, of which the former carries surfaces charges localized to $z=z_{\text {charge }}=-148.5 \AA$. Hence, the centers of the surface charges are placed $1.5 \AA$ outside the planar surface, and the surface charges protrude somewhat into the solution. The system is periodic in the $x$ and $y$ directions. Thus, we formally investigate the adsorption from a solution with a finite polyion concentration; however, we argue that the surface is sufficiently large to make the results representative for the adsorption of a single polyion. The model is illustrated in Figure 2.

Some complementary simulations with (i) a cubic lattice arrangement of the surface charges and (ii) surface charges below the wall have also been performed to examine the role of the order of surface charges and their protrusion into the solution.

All interactions are taken as pairwise additive. The total potential energy $U$ of the system can be expressed as a sum of four contributions according to

$$
U=U_{\text {nonbond }}+U_{\text {bond }}+U_{\text {ang }}+U_{\text {ext }}
$$

The nonbonded potential energy, $U_{\text {nonbond, }}$ is given by

$$
U_{\text {nonbond }}=\sum_{i<j} u_{i, j}\left(r_{i, j}\right)
$$

where the summation extends over polyion beads, surface charges, and simple ions with $u_{i, j}$ representing the electrostatic potential plus a hard-sphere repulsion according to

$$
u_{i, j}\left(r_{i, j}\right)= \begin{cases}\infty & r_{i, j}<R_{i}+R_{j} \\ \frac{Z_{i} Z_{j} e^{2}}{4 \pi \epsilon_{\mathrm{o}} \epsilon_{\mathrm{r}}} \frac{1}{r_{i, j}} & r_{i, j} \geq R_{i}+R_{j}\end{cases}
$$


where $Z_{i}$ is the valence of the particle $i, R_{i}$ the radius of the particle $i, r_{i, j}$ the distance between particles $i$ and $j, e$ the elementary charge, $\epsilon_{\mathrm{o}}$ the permittivity of vacuum, and $\epsilon_{\mathrm{r}}$ the relative permittivity of the solvent.

Polyion beads are connected by harmonic bonds, and the bond potential energy of the polyion, $U_{\text {bond }}$, becomes

$$
U_{\text {bond }}=\sum_{i=1}^{N_{\text {bead }}-1} \frac{k_{\text {bond }}}{2}\left(r_{i, i+1}-r_{0}\right)^{2}
$$

where $N_{\text {bead }}$ is the number of beads, $r_{i, i+1}$ is the distance between two connected beads with the equilibrium separation $r_{0}=5 \AA$, and the force constant $k_{\text {bond }}=0.4 \mathrm{~N} \mathrm{~m}^{-1}$. With the other interactions included, the typical root-mean-square (rms) beadbead separation becomes $\left\langle R_{\text {bead,bead }}{ }^{2}\right\rangle^{1 / 2} \approx 5.5 \AA$. The angular potential energy, $U_{\text {ang }}$, is given by

$$
U_{\text {ang }}=\sum_{i=1}^{N_{\text {bead }}-1} \frac{k_{\text {ang }}}{2}\left(\alpha_{i}-\alpha_{0}\right)^{2}
$$

where $\alpha_{i}$ is the angle formed by the vectors $\mathbf{r}_{i+1}-\mathbf{r}_{i}$ and $\mathbf{r}_{i-1}$ $-\mathbf{r}_{i}$ with the equilibrium angle $\alpha_{0}=180^{\circ}$ and the force constant $k_{\text {ang. }}$. by

Finally, the confining external potential energy, $U_{\text {ext }}$, is given

$$
U_{\mathrm{ext}}=\sum_{i} u_{\mathrm{ext}}\left(z_{i}\right)
$$

where the summation extends only over polyion beads and simple ions with

$$
u_{\text {ext }}\left(z_{i}\right)= \begin{cases}\infty & \left|z_{i}\right|>z_{\text {wall }} \\ 0 & \left|z_{i}\right|<z_{\text {wall }}\end{cases}
$$

Note that the electrostatic and hard-sphere interactions involving the surface charges are included in eqs 2 and 3.

For simplicity, the same hard-sphere radius $R_{i}=2 \AA$ has been used for the polyion beads, surface charges, and simple ions. Moreover, all charged species are monovalent. Throughout, $T=298 \mathrm{~K}$ and $\epsilon_{\mathrm{r}}=78.4$ have been used.

2.2. Systems. In our investigations, we have varied (i) the net charge of the surface $\Delta Z_{\text {surf }}$, (ii) the mobility of the surface charges (i.e., frozen vs mobile), and (iii) the intrinsic stiffness of the polyion regulated by $k_{\text {ang }}$ and expressed by its persistence length, $l_{\mathrm{p}} . N_{\text {bead }}=50$ was used throughout the work. The sum of the number of cations and anions is always 1000 (except for the crystal arrangement where 1058 was used), giving a hardsphere area fraction $1000 \pi R_{\text {bead }}^{2} / L_{x} L_{y}=0.31$, whereas the net surface charge $\Delta Z_{\text {surf }}$ is varied from +1000 to -20 . Two different flexibilities are considered, $l_{\mathrm{p}} \approx 7 \AA$ (flexible) and $l_{\mathrm{p}}$ $\approx 22 \AA$ (semiflexible) obtained by using $k_{\text {ang }}=0.0$ and $3.4 \times$ $10^{-24} \mathrm{~J} \mathrm{deg}^{-2}$, respectively.

The in-plane structure of the surface charges in the absence of the polyion (the unperturbed structure) has been analyzed using two-dimensional radial distribution functions. Such data for the net neutral surface are shown in Figure 3. A prominent maximum appears at the contact distance for the cation-anion pair. The local density of like-charged ions remains near the average one, even at short separation, as a consequence of the large hard-sphere area fraction. Finally, the spatial correlations extends to $\sim 12 \AA$.

2.3. Simulation Details. All Monte Carlo (MC) simulations were performed in the canonical ensemble employing the

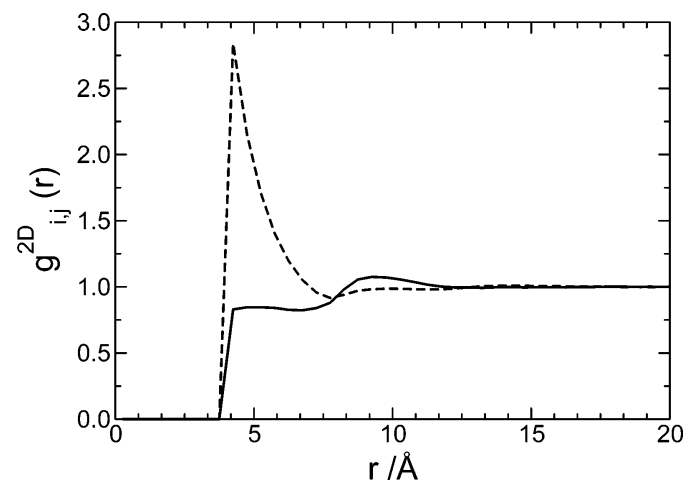

Figure 3. Two-dimensional radial distribution functions for like-like (solid curve) and like-unlike (dashed curve) surface charges for a net neutral surface $\left(\Delta Z_{\text {surf }}=0\right)$ in the absence of polyion.

standard Metropolis algorithm. ${ }^{37}$ The long-range electrostatic interactions were handled using the Ewald summation with a recent extension to slab geometry. ${ }^{38}$ Nevertheless, test simulations using the minimum image convention gave results essentially indistinguishable from those with the Ewald summation.

Three different types of MC trial moves were employed for the polyion: (i) single bead move, (ii) translation of the entire chain, and (iii) slithering move where one of the end beads is moved to the opposite end of the chain with biased radial and angular positioning. The single particle move was attempted 100 times more often than the other two types of moves. The simple ions were subjected to translational moves and the surface charges (when mobile) to translational moves restricted to the $x y$ plane.

In the case of frozen surface charges, equilibration of systems containing surface charges and the necessary amount of simple ions to ensure electroneutrality were performed. The final configurations of the surface charges were used in the subsequent simulations of systems containing a polyion with frozen surface charges. Hence, the surface charge configurations in these simulations thus possess a 2D liquidlike disordered structure.

Each simulation included an equilibration of at least $2 \times 10^{5}$ trial moves per particle followed by a production run of at least $5 \times 10^{5}$ trial moves per particle. Statistical uncertainties were evaluated by dividing the total simulation into subbatches. All the simulations were performed using the simulation package MOLSIM. ${ }^{39}$

2.4. Properties Examined. Properties describing (i) the average position of beads and simple ions, (ii) the spatial extension of the polyion, and (iii) the conformation of the polyion in relation to the surface have been used to characterize the adsorption of the polyion onto the surface. Polyion beads located within $8.5 \AA$ from the plane of the surface charges were considered to be adsorbed, and the number of adsorbed beads is denoted by $N_{\text {ads. }}$. The extension of the polyion was characterized by its radius of gyration. In particular, we have employed its projections onto the $x y$ plane (parallel to the surface) and the $z$ axis (perpendicular to the surface) according to

$$
\left\langle R_{\mathrm{G}_{x y}}{ }^{2}\right\rangle^{1 / 2}=\left\langle\left.\frac{1}{N_{\text {bead }}} \sum_{i=1}^{N_{\text {bead }}}\left[\left(x_{i}-x_{\mathrm{CM}}\right)^{2}+\left(y_{i}-y_{\mathrm{CM}}\right)^{2}\right]\right|^{1 / 2}\right.
$$




$$
\left\langle R_{\mathrm{G}_{z}}{ }^{2}\right\rangle^{1 / 2}=\left\langle\frac{1}{N_{\text {bead }}} \sum_{i=1}^{N_{\text {bead }}}\left(z_{i}-z_{\mathrm{CM}}\right)^{2}\right\rangle^{1 / 2}
$$

where the subindex CM refers to the center of mass of the polyion and $\langle\ldots\rangle$ to an ensemble average. Adsorbed chains have also been characterized by the number and length of their trains, loops, and tails. A train constitutes a sequence of adsorbed beads, a loop is a sequence of beads having both ends connected to trains, and a tail is a sequence of beads having exactly one end connected to a train. Of particular interest is the number of beads in tails, $N_{\text {tail }}$. In many cases, the adsorbed polyion possesses a single long tail, and the remaining part forms a sequence of trains and loops. In those cases, $N_{\text {tail }}$ will denote the length of the single tail, and the radius of gyration and its two components have been determined separately for the tail and the remaining part of the polyion containing the trains and the loops.

\section{Results and Discussion}

3.1. State of Adsorbed Polyion. We will start by considering the conditions at which the polyion is adsorbed and, in particular, investigate the state of the adsorbed polyion at the different conditions. First, the general dependence of the surface net charge will be discussed, and then, the effects of the surface charge mobility and the polyanion flexibility will be examined.

3.1.1. General. The number of adsorbed beads as a function of the surface net charge at different surface charge mobility and different polyion flexibility is shown in Figure 4a. At the two highest surface net charges investigated, $\Delta Z_{\text {surf }}=500$ and 1000 , the number of adsorbed beads, $N_{\text {ads }} \approx N_{\text {bead }}=50$, demonstrating a very strong adsorption. As $\Delta Z_{\text {surf }}$ is reduced, particularly below 50 , the number of adsorbed beads decreases. Nevertheless, even at a net neutral surface (i.e., $\Delta Z_{\text {surf }}=0$ ), the polyion is adsorbed; the number of adsorbing beads varies between 6 and 34. At $\Delta Z_{\text {surf }}=-20$, no adsorption occurs. The corresponding data for the number of beads residing in tails, $N_{\text {tail }}$, are displayed in Figure $4 \mathrm{~b}$. For $\Delta Z_{\text {surf }}>20$, only a few beads are located in tails, and by decreasing $\Delta Z_{\text {surf }}$, the number of beads in tails increases markedly. The number of beads residing in loops, $N_{\text {bead }}-N_{\text {ads }}-N_{\text {tail }}$, also typically increases with decreasing $\Delta Z_{\text {surf }}$ (data not shown).

The extensions of the polyion parallel and perpendicular to the surface have been quantified using rms radii of gyration projected onto the $x y$ plane and the $z$ axis, respectively. Figure 5a shows that the largest extension parallel to the surface $\left(2\left\langle R_{\mathrm{G}_{x y}}{ }^{2}\right\rangle^{1 / 2} \approx 80-140 \AA\right)$ appears at high surface net charge, and $\left\langle R_{\mathrm{G}_{x y}}{ }^{2}\right\rangle^{1 / 2}$ displays a moderate reduction as the adsorption becomes weaker. Figure $5 \mathrm{~b}$ shows that the extension of the polyion perpendicular to the surface plane $2\left\langle R_{\mathrm{G}_{z}}{ }^{2}\right\rangle^{1 / 2}$ is below $10 \AA$ for $\Delta Z_{\text {surf }}>20 \AA$; hence, the polyion is adsorbed in a flat arrangement. Not unexpectedly, $\left\langle R_{\mathrm{G}_{z}}{ }^{2}\right\rangle^{1 / 2}$ increases prominently as $\Delta Z_{\text {surf }}$ approaches zero.

A more detailed account of the adsorbed state is given in Figure 6. Here, different properties are given as a function of the number of adsorbed beads with the net surface charge as an implicit parameter. Figure 6a displays the number of loops. At large $N_{\text {ads }}(\sim 50)$, obtained for high $\Delta Z_{\text {surf }}$, there is no or, at most, one loop. At decreasing $\Delta Z_{\text {surf }}$ with the concomitantly smaller $N_{\text {ads }}$, the number of loops first increases, reaches a maximum, and then drops. The decrease at small $N_{\text {ads }}$ is a consequence of the small part of the polyion being anchored to the surface, which eventually, by necessity, cannot accommodate any loops. The rms radius of gyration perpendicular to the surface plane averaged over the loop and train beads is shown
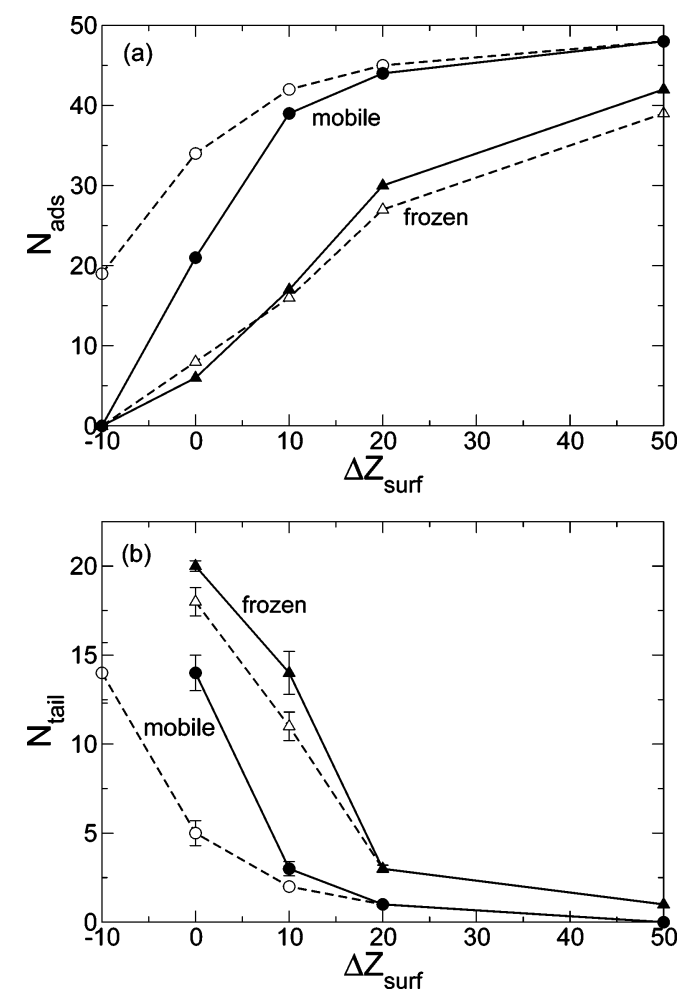

Figure 4. (a) Number of adsorbed beads and (b) number of beads residing in tails vs the surface net charge for a system with frozen (triangles) or mobile (circles) surface charges and a flexible (open symbols and dashed lines) or semiflexible (filled symbols and solid lines) polyanion. Uncertainty bars are given.
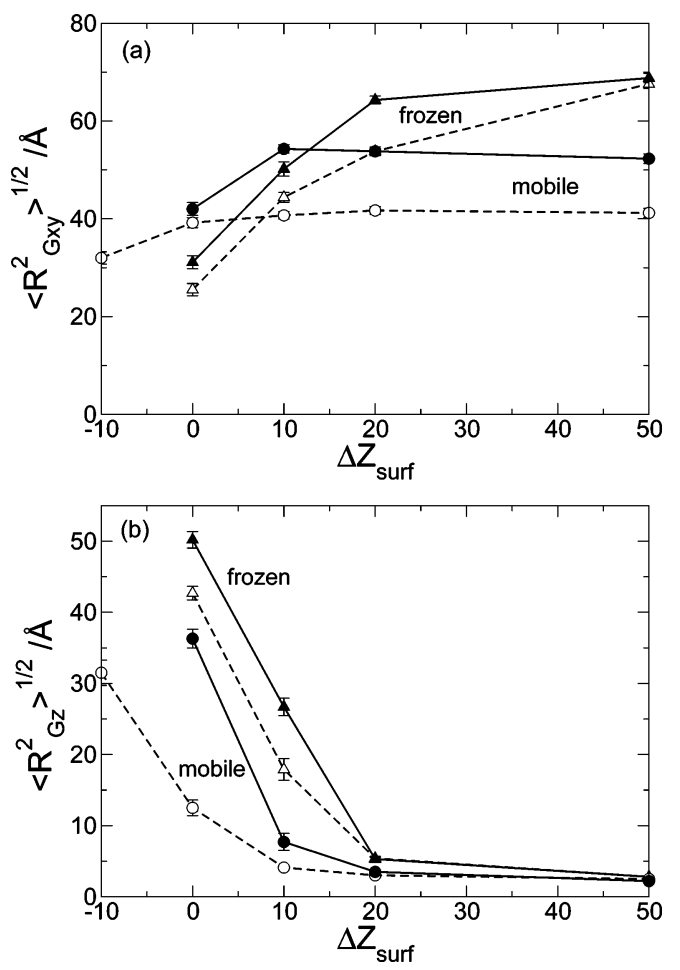

Figure 5. Rms radius of gyration of the polyion projected onto (a) the surface plane and (b) the surface normal vs the surface net charge for a system with frozen (triangles) or mobile (circles) surface charges and a flexible (open symbols and dashed lines) or semiflexible (filled symbols and solid lines) polyanion. Uncertainty bars are given.

in Figure 6b. It is seen that as the number of adsorbed beads is decreased the thickness of this layer is first slightly increased and then reduced. Thus, when the loops are more numerous, 

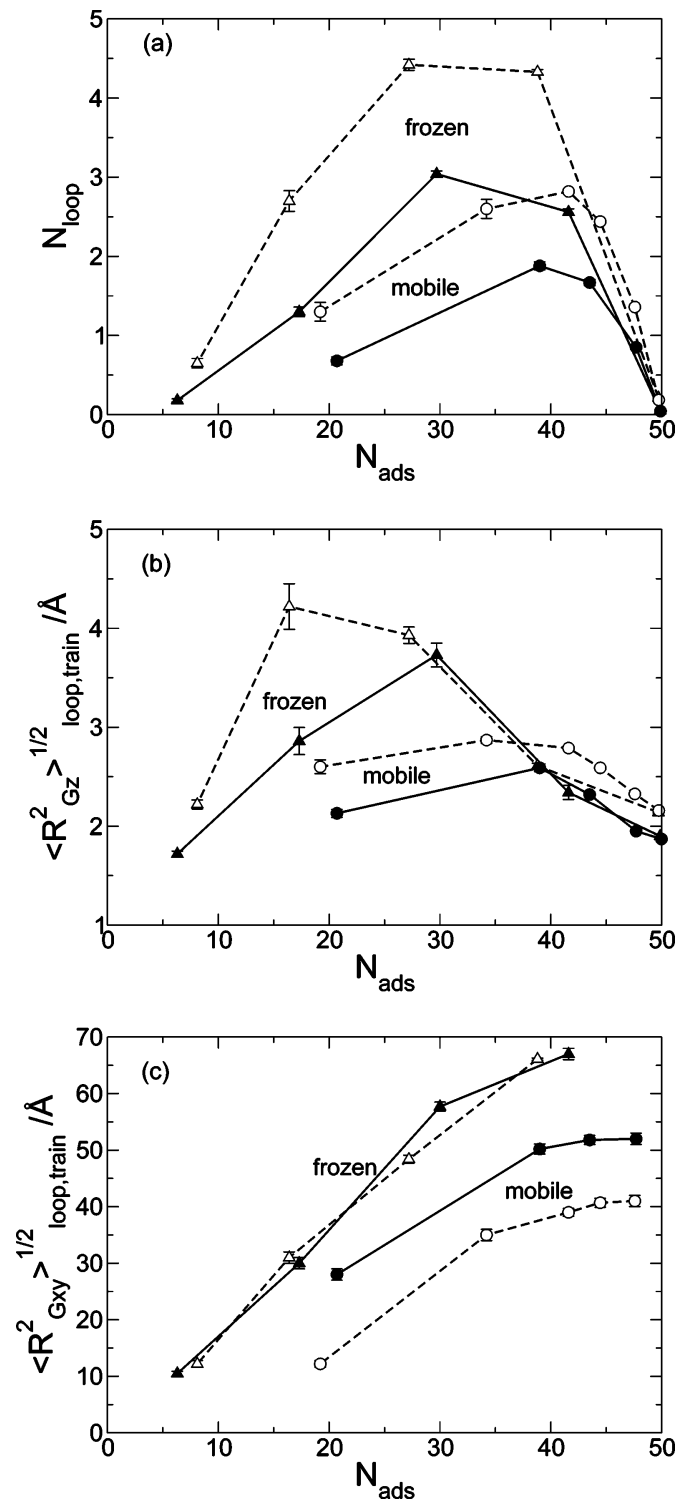

Figure 6. (a) Number of loops, (b) rms radius of gyration based on train and loop beads projected onto the surface normal, and (c) rms radius of gyration based on train and loop beads projected onto the surface plane vs the number of adsorbed polyion beads for a system with frozen (triangles) or mobile (circles) surface charges and a flexible (open symbols and dashed lines) or semiflexible (filled symbols and solid lines) polyanion. Uncertainty bars are given.

they also extend further away from the surface. However, the thickness is generally small; thus, the loops do not extend far from the surface. Finally, Figure $6 \mathrm{c}$ shows the rms radius of gyration parallel to the surface plane averaged over the loop and train beads. At large $N_{\text {ads }}(\sim 50)$, the extension parallel to the surface ranges from 40 to $70 \AA$. At decreasing $N_{\text {ads }}$, $\left\langle R_{\mathrm{G}_{x y}}{ }^{2}\right\rangle_{\text {loop,train }}^{1 / 2}$ reduces continuously and approaches ca. $10 \AA$, before the polyion ceases to be adsorbed.

Hence, the model reproduces the conventional picture that a charged polyion is (i) adsorbed in an extended conformation parallel to the surface at a highly and oppositely charged surface and (ii) only partly adsorbed with extended tail(s) at a weakly charged surface. At the latter condition, extended loops are typically present as well, but the short contour length of the polyion here suppresses them. A noticeable observation, however, is the adsorption of the polyanion onto a net neutral, or even a weakly negatively charged, surface; a feature not appearing in mean-field theories or in models with homogeneous surface charge densities.
3.1.2. Dependence on Surface Charge Mobility. The influence of the fluidity of the surface charges on the adsorption will now be examined. This will be pursued by comparing corresponding data for frozen (triangles in Figures 4-6) and mobile (circles in Figures 4-6) surface charges.

For highly charged surfaces, $\Delta Z_{\text {surf }} \geq 500$, there is no difference between the two types of surfaces (data not shown). However, Figure $4 \mathrm{a}$ shows that at a lower $\Delta Z_{\text {surf }}$ the number of adsorbed beads reduces more slowly for mobile surface charges; hence, the adsorption is stronger with mobile surface charges. Concomitantly, for a given value of $\Delta Z_{\text {surf }}$, the tail contains fewer beads for the surface with mobile surface charges as demonstrated in Figure 4b.

Regarding the extension parallel to the surface, Figure 5a shows that generally the polyion becomes less stretched when adsorbed at a surface with mobile charges as compared to a surface with frozen ones. However, an exception appears at conditions where the polyion is weakly adsorbed $\left(\Delta Z_{\text {surf }} \leq\right.$ 10): here, the opposite appears. The differences in the extension perpendicular to the surface between frozen and mobile surface charges given in Figure 5b follow closely those of the tail length.

The results of the more detailed investigation show, at a given number of adsorbed beads: (i) smaller number of loops (Figure 6a), (ii) higher vertical extension of the loop and train sections at high $N_{\text {ads }}$ but lower extension at lower $N_{\text {ads }}$ (Figure 6b), and (iii) smaller extension of the loop and train sections parallel to the surface plane (Figure 6c) for mobile surface charges as compared to frozen surface charges. Thus, for a comparison with the same $N_{\text {ads }}$, surface charge mobility leads to a reduction of the number of loops and of the vertical extension as well as to a more compact in-plane polyion conformation.

The complementary simulations with a cubic lattice arrangement of the surface charges at $\Delta Z_{\text {surf }}=18$ displayed only a weak bead adsorption, $N_{\text {ads }}=7$, and at $\Delta Z_{\text {surf }}=0$ no adsorption at all for both polyion flexibilities. From a comparison between these findings and corresponding data for frozen and mobile surface charges, we conclude that a disordered surface charge distribution strongly enhances the adsorption at weakly netcharged surfaces.

Another set of complementary simulations was made with surface charges localized in the plane $z_{\text {charge }}=-152 \AA$ and hence not protruding into the solution. At $\Delta Z_{\text {surf }}=0, N_{\text {ads }}$ remained the same (mobile surface charges), or no adsorption appeared (frozen surface charges) for both polyion flexibilities. Hence, protruding surface charges seem to enhance the adsorption, but the effect appears to be limited.

In summary, surface charge heterogeneity enhances polyion adsorption which is, furthermore, promoted by surface charge mobility. Surface charge protrusion also promotes the adsorption; however, this effect appears to be limited and has been examined in less detail.

3.1.3. Dependence on Polyion Flexibility. We have now arrived at the stage where the differences in the adsorption characteristics between the flexible (dashed lines in Figures 4-6) and semiflexible (solid lines in Figures 4-6) polyions will be assessed. Of course, the comparison between the two polyions will be made for the same surface charge mobility.

In terms of the adsorbed amount of beads on the frozen surface, for relatively high $\Delta Z_{\text {surf }}$, Figure 4a shows that $N_{\text {ads }}$ is larger for the semiflexible polyion as compared to the flexible one. Hence, the adsorption of the flexible polyion is weaker, an effect traditionally attributed to the larger loss of conformational entropy. However, for weakly adsorbed polyions appearing at $\Delta Z_{\text {surf }} \leq 10$ with $N_{\text {ads }} \leq 15$, the situation becomes the 
opposite, and the flexible polyanion displays a slightly larger number of adsorbed beads. Regarding the case with mobile surface charges, this "reverse" situation appears over a larger range of surface net charge $\Delta Z_{\text {surf }} \leq 20$ and for a larger range of number of adsorbed beads, $N_{\text {ads }} \leq 45$. In fact, the negatively charged flexible polyion remains adsorbed on a net negatively charged $\left(\Delta Z_{\text {surf }}=-10\right)$ surface. At the three other conditions, $N_{\text {ads }}=0$ at $\Delta Z_{\text {surf }}=-10$. Of course, we do not exclude the possibility that the polyanion adsorbs on net negatively charged surfaces in these cases as well; further simulations at $-10<$ $\Delta Z_{\text {surf }}<0$ are required to elucidate that. It is also seen in Figure $4 \mathrm{~b}$ that at moderate to weak adsorption $\left(\Delta Z_{\text {surf }} \leq 20\right)$ the semiflexible polyion possesses tails with more beads.

Figure 5a shows that the extension parallel to the surface is larger for the semiflexible polyion as compared to the flexible one at all conditions. Similarly, Figure 5b shows that the extension perpendicular to the surface also is larger for the semiflexible polyion.

The more detailed analysis shows that, for a given number of adsorbed beads, the semiflexible polyion presents: (i) a smaller number of loops (Figure 6a), (ii) a somewhat smaller vertical extension of the layer of trains and loops (Figure 6b), and (iii) a larger (mobile surface charges) or similar (frozen surface charges) extension parallel to the surface of the layer of trains and loops (Figure 6c) as compared to the flexible one. Hence, the increased stiffness of the polyion leads to fewer loops and a less vertically extended adsorbed layer and to a larger extension parallel to the surface plane with mobile surface charges.

3.2. Density Distributions. The distribution of the polyion beads and the simple ions perpendicular to the surface has been analyzed. Figure 7 provides the number densities on a logarithmic scale versus the $z$ coordinate at zero net surface charge for the four combinations of frozen and mobile surface charges with flexible and semiflexible polyions.

For all the considered systems, $\rho_{\text {bead }}(z)$ displays two regions: (i) a relatively high-density region close to the surface with a maximum ca. $5 \AA$ from the hard wall and (ii) an extended region with a nearly constant density (one exception) starting from $z$ $\approx-130 \AA$. The former region is narrow with a width between 8 and $15 \AA$, consistent with a surface layer composed only of trains and short loops, whereas the latter region originates, of course, from the stretched tail.

The maximal value of $\rho_{\text {bead }}(z)$ for the case of frozen surface charges (Figure 7a) is ca. $1 / 4$ of that for the case of mobile surface charges (Figure 7b), consistent with the different values of $N_{\text {ads }}$ (Figure $4 \mathrm{a}$ ). For both types of surfaces, the flexible polyion displays a larger maximal value, consistent with the larger number of adsorbed beads (Figure 4a).

The values of $\rho_{\text {bead }}(z)$ in the tail region are similar and exceed only slightly $1 /\left(L_{y} L_{z}\left\langle R_{\text {bead,bead }^{2}}\right\rangle^{1 / 2}\right)=5 \times 10^{-6} \AA^{-3}$ for a straight tail perpendicular to the surface. The case of mobile surface charges and the flexible chain constitutes an exception with a considerably thinner tail region, consistent with the much shorter tail, $N_{\text {tail }}=5$ (see Figure $4 \mathrm{~b}$ ). In the case with the frozen surface charges and extended tails, $\rho_{\text {bead }}(z)$ displays a local maximum at the tail end, implying a locally more folded structure at the tail end.

The corresponding simple ion density $\rho_{\text {ion }}(z)$ displays a similar behavior; however, its distribution is smoother for entropic reasons. In particular, $\rho_{\text {ion }}(z) \ll \rho_{\text {bead }}(z)$ close to the surface, and $\rho_{\text {ion }}(z) \gg \rho_{\text {bead }}(z)$ far from the surface, with $\rho_{\text {ion }}(z) \approx \rho_{\text {bead }}(z)$ at intermediate distances (again, the short tail case constitutes an exception).
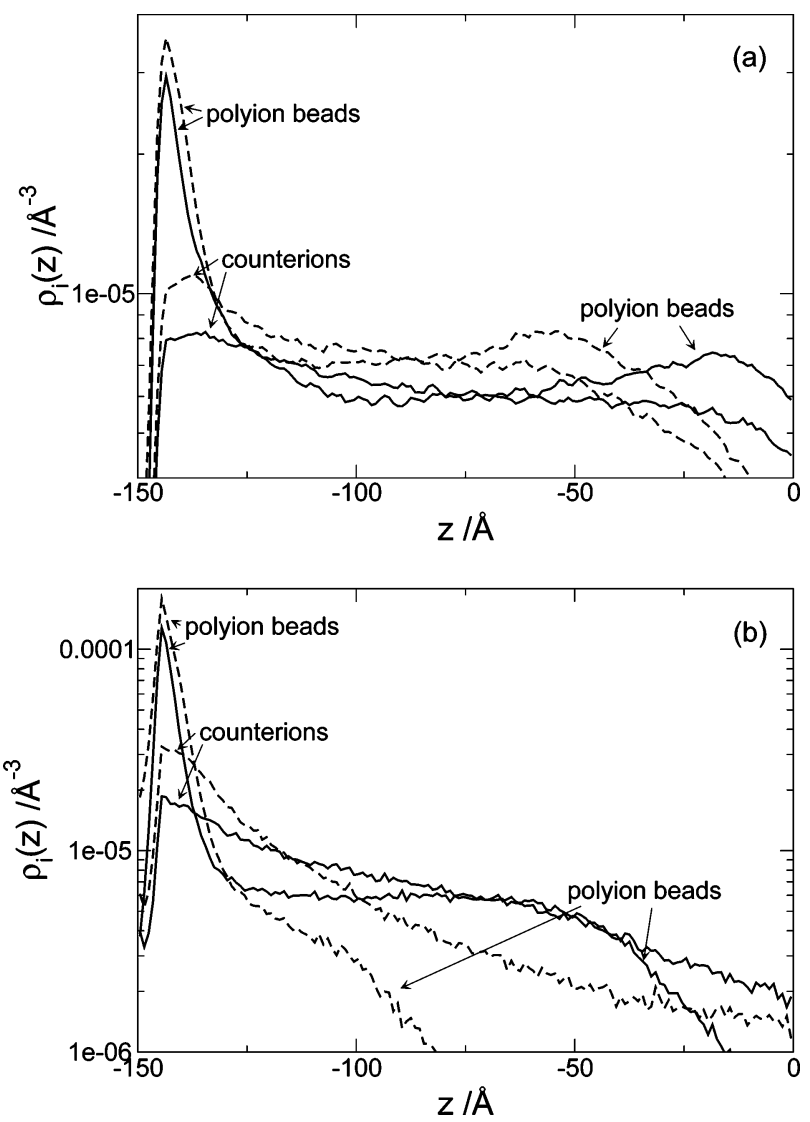

Figure 7. Number density of polyion beads and polyion counterions vs the $z$-coordinate for a system with (a) frozen and (b) mobile surface charges and flexible (dashed curves) or semiflexible (solid curves) polyion at zero surface net charge.

Close to the surfaces, $\rho_{\text {bead }}(z)$ and $\rho_{\text {ion }}(z)$ are reduced because of hard-sphere overlap with the protruding surface groups, and $\rho_{\text {bead }}(z)$ is also reduced because of loss of conformational entropy. The maximum in $\rho_{\text {bead }}(z)$ appears approximately at $z$ $=-144.5 \AA$, which is the largest $z$ coordinate where hard-sphere contacts may appear between beads and surface groups. Moreover, $\rho_{\text {ion }}(z)$ displays a maximum (frozen surface charges) or a cusp (mobile surface charges) approximately at $z=-144.5$ $\AA$. We also notice that the bead density at $z=z_{\text {wall }}=-150.0$ $\AA$ is largest for the flexible polyion with mobile surface charges $\left(2 \times 10^{-5} \AA^{-3}\right)$ and smallest for the semiflexible polyion with frozen surface charges $\left(9 \times 10^{-7} \AA^{-3}\right)$. Hence, the penetration of the polyion beads into the surface charge layer differs strongly among the conditions considered. The variation of the counterion contact density is much less.

3.3. Surface Polarization. The ability of the polyanion to adsorb to a net neutral surface is of course related to the presence of both negative and positive surface charges. In the case of frozen surface charges in a disordered and liquidlike configuration, there are regions with a surplus of cations at which the polyion is adsorbed. Previous studies have examined that in some detail. ${ }^{32,33,36}$ However, less investigated is the spatial polarization of the surface charges by the presence of a polyion. This polarization has been analyzed in two different ways; both approaches are based on a division of the $200 \times 200 \AA^{2}$ surface into $10020 \times 20 \AA^{2}$ squares.

In the first approach, the normalized probability function of the net charge $\Delta Z_{\mathrm{sq}}$ of such a square was determined. On average, there will be 10 charges in a square. Figure 8a shows this probability function for a system with a surface with zero net charge without polyion, $P_{0}\left(\Delta Z_{\mathrm{sq}}\right)$. As expected, the prob- 


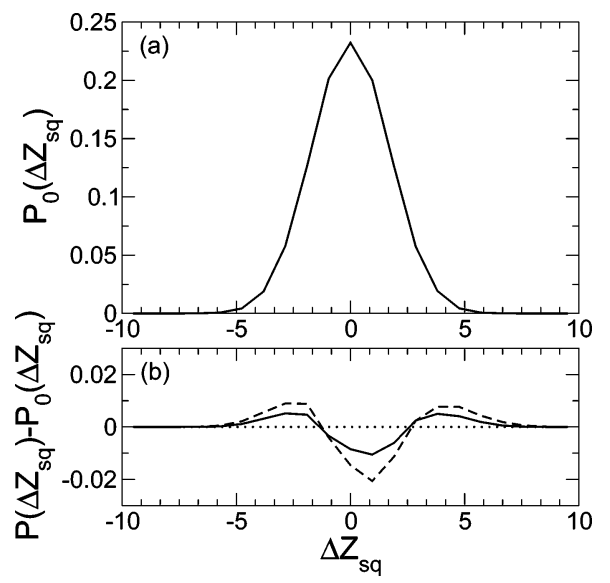

Figure 8. (a) Probability distribution of the net charge of $20 \times 20 \AA^{2}$ squares of the net neutral catanionic surface in the absence of the polyion and (b) the difference in the probability distribution of the net charge between a system with a flexible (dashed line) or semiflexible (solid line) polyion and without polyion.

ability function is symmetric at $\Delta Z_{\mathrm{sq}}=0$. Moreover, the function decays rapidly for both negative and positive values, and it is practically zero at $\left|\Delta Z_{\mathrm{sq}}\right|>5$, half of its maximal absolute value of 10 . Figure $8 \mathrm{~b}$ shows the change in the probability function when a flexible or semiflexible polyion is adsorbed. The presence of the polyion causes an increase in the probability of regions with a high positive charge (from +3 to +7 ) and moderate negative charge $(-1$ to -5$)$ while a decrease in the probability of regions with near zero net charge. The effect is almost twice as large for the flexible polyion. Although the changes may appear small, it should be recalled that the examined polarization is smeared out over the whole surface. The effect observed arises from a polyion possessing 50 positive monovalent charges adsorbed onto a surface comprising 500 positive and 500 negative charges.

The other approach focuses on the magnitude of the surface polarization near a polyion and how the polarization decreases away from it. Figure 9a shows a contour diagram of the surface polarization based on the average polarization in each of the 100 squares determined for a fixed polyion conformation. The average polarizations were obtained from a separate $\mathrm{MC}$ simulation providing Boltzmann-weighted positions of the surface charges for a fixed and representative polyion conformation displayed in Figure 9b (top view) and c (perspective view). Figure $9 \mathrm{~b}$ also provides a picture of the liquidlike surface charge structure.

Most surfaces display an average charge density close to zero with a few slightly negatively charged squares with $\left\langle\Delta Z_{\mathrm{sq}}\right\rangle$ down to -0.4 . However, in the vicinity of the polyion, we have $\left\langle\Delta Z_{\mathrm{sq}}\right\rangle$ $>0$ (see Figure 9a). Near the right end of the polyion, a region with a very positive polarization $(+6)$ is observed, corresponding to a sequence of folded and firmly adsorbed beads (see Figure 9c). Near the left end of the polyion, two neighboring regions with high positive polarization appear $(+4)$. In Figure 9c, a loop joining the right and left regions of high positive surface polarization is clearly visible. Moreover, Figure 9a shows that the surface polarization has vanished $\sim 15 \AA$ away from the polyion. Thus, the charges of the polyion considerably polarize the fluid catanionic surface, and the polarization is fairly local, making the polarization pattern an image of the vertical distance between the polyion beads and surface.

Finally, the energetics of the polarization have been determined. As expected, the polarization leads to an increase in the average electrostatic potential energy among the surface charges. (a)

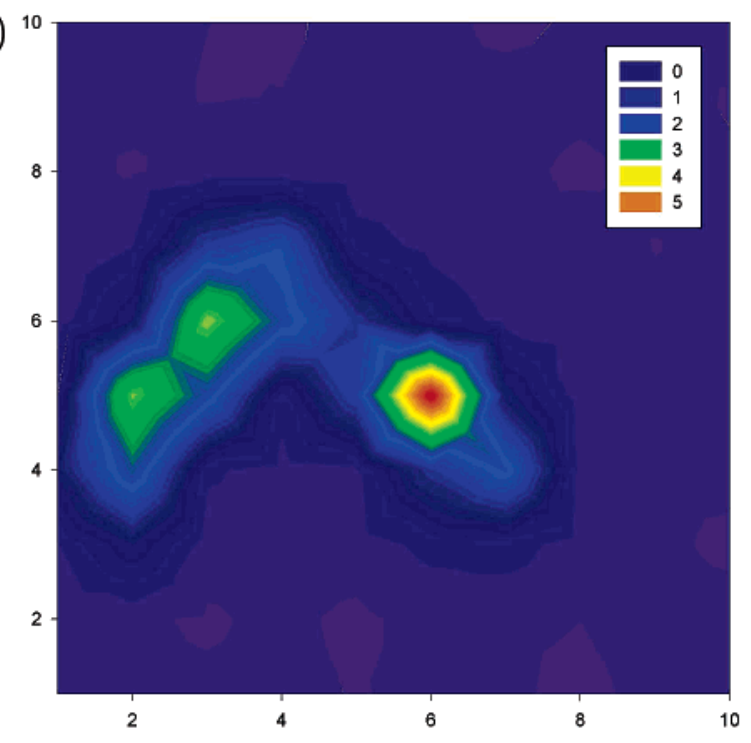

(b)

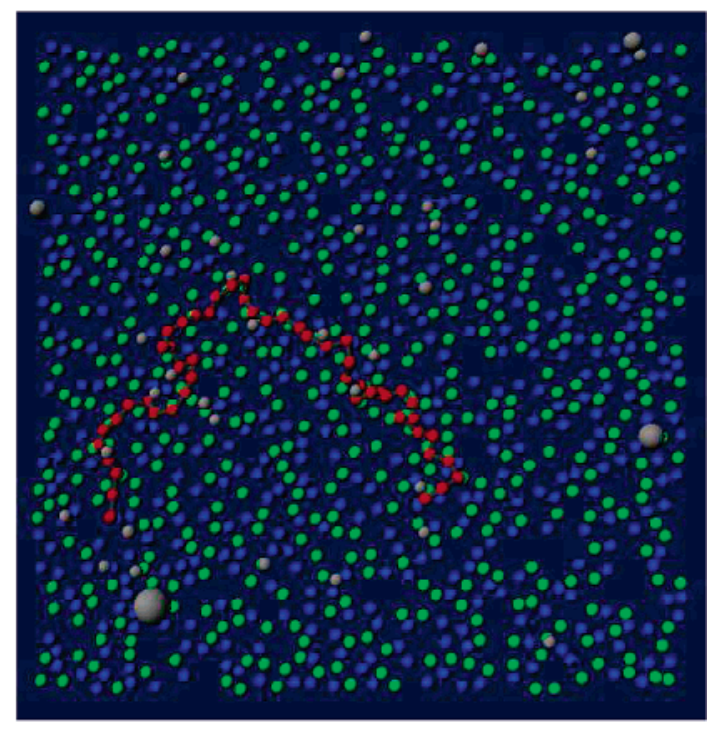

(c)

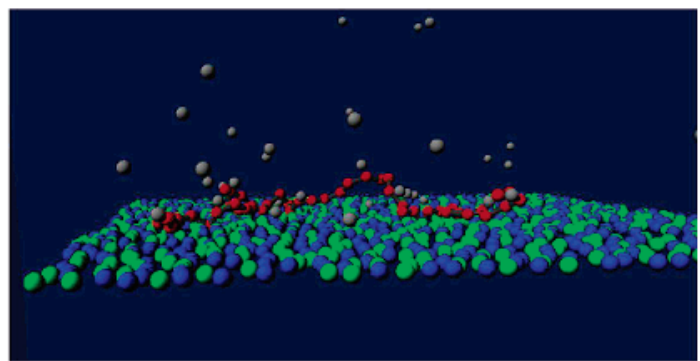

Figure 9. (a) Contour plot of the average net charge of $20 \times 20 \AA^{2}$ squares of a net neutral catanionic surface with mobile surface charges in the presence of an adsorbed flexible polyion in a fixed conformation and its counterions, (b) representative snapshot, top view, and (c) same snapshot, perspective view. In (a), the average net charge of the squares was obtained from Monte Carlo simulations with a fixed polyion configuration. The color code is given for a selected number of contour intervals.

However, the increased attractive electrostatic surface-polyion potential energy exceeds this polarization penalty. It was found that both contributions are nearly proportional to the number of adsorbed polyion beads.

\section{Conclusions}

On the basis of Monte Carlo simulations of a simple model comprising a spring-bead chain representing a negatively 
charged polyion and a surface carrying both negative and positive charges representing a surface of a charged membrane, we have found that the details of the membrane surface significantly influence the polyion adsorption. In fact, these details have a stronger effect on the adsorption than the polyion flexibility does. The main findings are as follows:

(A) With a large excess of positive surface charges, the classical picture of a strongly adsorbed polyion with an extended and flat configuration emerged.

(B) Adsorption appeared also at a net neutral surface or at a weakly negatively charged surface. A disordered surface charge distribution was found necessary for this behavior. An enhanced adsorption was observed for mobile surface charges.

(C) At weak adsorption, a flexible chain adsorbed more strongly as compared to a semiflexible one.

(D) Protruding surface charges slightly promoted adsorption, but the effects were weak and not examined in detail.

(E) Findings (B) and (C) go beyond the conventional adsorption behavior of a polyion at a homogeneously charged surface. (i) Regarding the case with frozen surface charges, the reason is an adsorption of the polyion onto net positively charged regions (patches). The ability to adsorb onto these irregularly positioned patches increases with increasing chain flexibility. (ii) The enhanced adsorption with mobile surface charges originates from the spatial polarization of the surface charges. A flexible chain is able to fold into a more compact in-plane configuration and hence induces a larger polarization. The net effect of the polarization is an additional attractive potential energy component. The polarization is local and constitutes an image of the polyion conformation.

Hence, the increased complexity of the representation of a charged membrane surface alters two classical aspects of the adsorption of polyions to charged surfaces. In this work, we have focused on salt-free systems. Adding an electrolyte would result in a screening of the electrostatic interactions and generally would decrease the attraction and adsorption of the polyion to the surface; however, we believe that the main conclusions of this work should not be affected by a moderate amount of salt. Furthermore, we have so far not included the ability of the surface charges to individually protrude differently into the solution or taken into account the lower relative permeability of the interior of the membrane, two other aspects also affecting the polyion adsorption.

Acknowledgment. This work was supported by grants from an EU Research Training Network, CIPSNAC (contract no. MRTN-CT-2003-504932), and the Fundação para a Ciência e Tecnologia (FCT) (POCTI/QUI/45344/2002, and POCTI/QUI/ 45331/2002).

\section{References and Notes}

(1) Felgner, P. L.; Gadek, T. R.; Holm, M.; Roman, R.; Chan, H. W.; Wenz, M.; Northrop, J. P.; Ringold, G. M.; Danielsen, M. Proc. Natl. Acad. Sci. U.S.A. 1987, 84, 7413-7418.

(2) Lasic, D. Liposomes in Gene Delivery; CRC Press: Boca Raton, FL, 1997.
(3) Barreleiro, P. C. A.; May, R. P.; Lindman, B. Faraday Discuss. 2003, 122, 191-201.

(4) Barreleiro, P. C. A.; Lindman, B. J. Chem. Phys. B 2003, 107, $6208-6213$

(5) Rädler, J. O.; Koltover, I.; Salditt, T.; Safinya, C. R. Science 1997, $275,810-814$.

(6) Rädler, J. O.; Koltover, I.; Jamieson, A.; Salditt, T.; Safinya, C. R. Langmuir 1998, 14, 4272-4283.

(7) Lasic, D. D.; Strey, H.; Stuart, M. C. A.; Podgornik, R.; Frederik, P. M. J. Am. Chem. Soc. 1997, 119, 832-833.

(8) Dias, R. S.; Lindman, B.; Miguel, M. J. Phys. Chem. B 2002, 106, 12600-12607.

(9) Fenske, D. B.; MacLachlan, I.; Cullis, P. R. Curr. Opin. Mol. Ther. 2001, 3, 153-158.

(10) Ilies, M. A.; Balaban, A. T. Expert Opin. Ther. Pat. 2001, 11, $1729-1752$

(11) de Lima, M. C. P.; Neves, S.; Filipe, A.; Duzgunes, N.; Simões, S. Curr. Med. Chem. 2003, 10, 1221-1231.

(12) Eskilsson, K.; Leal, C.; Lindman, B.; Miguel, M.; Nylander, T. Langmuir 2001, 17, 1666-1669.

(13) Cardénas, M.; Braem, A.; Nylander, T.; Lindman, B. Langmuir 2003, 19, 7712-7718.

(14) Leonenko, Z. V.; Cramb, D. T. Nano Lett. 2002, 2, 305-309.

(15) Leonenko, Z. V.; Merkle, D.; Lees-Miller, S. P.; Cramb, D. T. Langmuir 2002, 18, 4873-4884.

(16) Schounten, S.; Stroeve, P.; Longo, M. L. Langmuir 1999, 15, 81338139 .

(17) Maier, B.; Rädler, J. O. Phys. Rev. Lett. 1999, 82, 1911-1913.

(18) Maier, B.; Rädler, J. O. Macromolecules 2000, 33, 7185-7194.

(19) Marques, E. F.; Regev, O.; Khan, A.; Miguel, M. G.; Lindman, B. Macromolecules 1999, 32, 6626-6637.

(20) Ashbaugh, H. S.; Boon, K.; Prud'homme, R. K. Colloid Polym. Sci. 2002, 280, 783-788.

(21) Antunes, F. E.; Marques, E. F.; Gomes, R.; Thuresson, K.; Lindman, B.; Miguel, M. G. Langmuir 2004, 20, 4647-4656.

(22) Regev, O.; Marques, E. F.; Khan, A. Langmuir 1999, 15, 642645.

(23) Mel'nikov, S. M.; Dias, R.; Mel'nikova, Y. S.; Marques, E. F.; Miguel, M. G.; Lindman, B. FEBS Lett. 1999, 453, 113-118.

(24) Denisov, G.; Wanaski, S.; Luan, P.; Glaser, M.; McLaughlin, S. Biophys. J. 1998, 74, 731-744.

(25) Harries, D.; May, S.; Ben-Shaul, A. Colloids Surf., A 2002, 208, $41-50$.

(26) Fleck, C.; Netz, R. R.; Hennig, H.; von Grünberg, H. Biophys. J. 2002, 82, 76-92.

(27) Clausen-Schaumann, H.; Gaub, H. E. Langmuir 1999, 15, 82468251

(28) Fleer, G. J.; Stuart, M. A. C.; Scheutjens, J. M. H. M.; Cosgrove, T.; Vincent, B. Polymers at interfaces; Chapman \& Hall: London, 1993.

(29) Andelman, D.; Joanny, J.-F. C. R. Acad. Sci., Ser. IV: Phys. Astrophys. 2000, 1, 1153-1162.

(30) Netz, R. R.; Andelman, D. Phys. Rep. 2003, 380, 1-95.

(31) Gottstein, W.; Kreitmeier, S.; Wittkop, M.; Göritz, D.; Gotsis, F. Polymer 1997, 38, 1607-1613.

(32) Ellis, M.; Kong, C. Y.; Muthukumar, M. J. Chem. Phys. 2000, 112 , 8723-8729.

(33) McNamara, J.; Kong, C. Y.; Muthukumar, M. J. Chem. Phys. 2002, $117,5354-5360$.

(34) Bruinsma, R.; Mashl, J. Europhys. Lett. 1998, 41, 165-170.

(35) May, S.; Harries, D.; Ben-Shaul, A. Biophys. J. 2000, 79, 17471760

(36) de Vries, R.; Weinbreck, F.; de Kruif, C. G. J. Chem. Phys. 2003, $118,4649-4659$.

(37) Allen, M. P.; Tildesley, D. J. Computer simulation of liquids; Clarendon: Oxford, 1987.

(38) Arnold, A.; de Joannis, J.; Holm, C. J. Chem. Phys. 2002, 117, $2496-2502$.

(39) Linse, P. MOLSIM, version 3.1.7; 2001 